\title{
A new angiographic imaging platform reduces radiation exposure for patients with liver cancer treated with transarterial chemoembolization
}

\author{
Ruediger E. Schernthaner ${ }^{1} \cdot$ Rafael Duran $^{1} \cdot$ Julius Chapiro $^{1} \cdot$ Zhijun Wang $^{1}$ • \\ Jean-Françcois H Geschwind ${ }^{1}$ • MingDe Lin $^{2}$
}

Received: 17 October 2014 / Revised: 15 March 2015 / Accepted: 16 March 2015 / Published online: 9 May 2015

(C) The Author(s) 2015. This article is published with open access at Springerlink.com

\begin{abstract}
Objectives To quantify the reduction of radiation liver cancer patients are exposed to during transarterial chemoembolization (TACE), while maintaining diagnostic image quality, using a new $\mathrm{C}$-arm imaging platform.

Methods In this prospective, HIPAA-compliant, IRB-approved, two-arm trial, 78 consecutive patients with primary or secondary liver cancer were treated with TACE on a C-arm imaging platform before and after an upgrade incorporating optimized acquisition parameters and advanced real-time image processing algorithms. Dose area product (DAP) and radiation time of each digital fluoroscopy (DF), digital subtraction angiography (DSA) and cone beam CT (CBCT) were recorded. DSA image quality was assessed by two blinded and independent readers on a four-rank scale.

Results Both cohorts showed no significant differences with regard to patient characteristics and tumour burden. The new system resulted in a statistically significant reduction of cumulative DAP of $66 \%$ compared to the old platform (median 132.9 vs. $395.8 \mathrm{~Gy} \mathrm{~cm}^{2}$ ). Individually, DAP of DF, DSA and CBCT decreased by $52 \%, 79 \%$ and $15 \%(p<0.01, p<0.01$, $p=0.51$ ), respectively. No statistically significant differences
\end{abstract}

Electronic supplementary material The online version of this article (doi:10.1007/s00330-015-3717-0) contains supplementary material, which is available to authorized users.

Jean-François H Geschwind

jfg@jhmi.edu

1 Russell H. Morgan Department of Radiology and Radiological Science, Division of Vascular and Interventional Radiology, The Johns Hopkins Hospital, Sheikh Zayed Tower, Suite 7203, 1800 Orleans St, Baltimore, MD 21287, USA

2 Ultrasound Imaging and and Interventions (UII), Philips Research North America, Briarcliff Manor, NY, USA in DSA image quality were found between the two imaging platforms.

Conclusions The new imaging platform significantly reduced radiation exposure for TACE procedures without increased radiation time or negative impact on DSA image quality.

Key Points

- The new C-arm system allowed reduction of radiation exposure by two thirds

- The procedure's course was not affected by the new platform

- No decrease in DSA image quality was observed after the radiation reduction

Keywords Interventional radiology · Therapeutic chemoembolization $\cdot$ Liver neoplasms $\cdot$ Radiation dosage Cone-beam computed tomography

\section{Introduction}

Since the 1950s, the numbers of diagnostic radiographic studies and image-guided interventional procedures have steadily increased [1]. In 2004, these diagnostic and therapeutic studies were the largest source of man-made radiation exposure and contributed to about $14 \%$ of the total worldwide exposure from man-made and natural sources [2]. The consequences of radiation, described as deterministic and stochastic effects, are of major concern [3, 4]. Following the "as low as reasonably achievable" (ALARA) principle, there is a balance between dose reduction and maintaining image quality at a diagnostic level [5].

Transarterial chemoembolization (TACE) has become an important treatment option for a number of primary and secondary liver malignancies [6-10]. In most patients, TACE is performed repeatedly because of the multifocal nature of the disease, incompletely treated lesions or disease recurrence. 
This exposes both patients and clinical staff to high doses of ionizing radiation [11].

A new X-ray imaging platform (AlluraClarity; Philips Healthcare, Best, Netherlands) which incorporates optimized acquisition parameters and several real-time image processing algorithms with the goal to reduce radiation exposure while maintaining diagnostic image quality was recently made available. In the scope of diagnostic and interventional neuroradiological procedures, this new platform showed a significant radiation exposure reduction of $60 \%$ for the whole procedure and of $75 \%$ for digital subtraction angiography (DSA) without a decrease in image quality $[12,13]$. In comparison to neuroradiological procedures, TACE requires a higher tube current due to the larger field of view and body volume that needs to be passed by the X-ray photons, resulting in a higher radiation exposure in the same amount of time. In addition, abdominal procedures are more prone to motion artefacts, which could limit the applicability of the real-time image processing algorithms of the new imaging platform. Furthermore, the previously mentioned neuroradiological studies evaluated only two-dimensional imaging (digital fluoroscopy (DF) and DSA), and no three-dimensional imaging, such as cone beam computed tomography (CBCT), which is often performed during TACE procedures [14].

The purpose of our study was to quantify the reduction of radiation that liver cancer patients are exposed to during transarterial chemoembolization (TACE) while maintaining diagnostic image quality, using a new $\mathrm{C}$-arm imaging platform.

\section{Materials and methods}

\section{Study cohort}

This prospective, single-institution, two-arm study was conducted in compliance with the Health Insurance Portability and Accountability Act and was approved by the institutional review board. In January and February 2014, 26 consecutive patients with primary or secondary liver cancer treated with TACE at our institution using the C-arm system (Allura Xper FD20, Philips Healthcare, Best, the Netherlands) available at that time were included (control group). The imaging platform was then upgraded to the new platform (AlluraClarity) and a second cohort of 52 consecutive patients between March and June 2014 with liver cancer treated with TACE (study group) was included. Thus the final population included a total of 78 patients.

\section{MR imaging technique}

All patients underwent baseline MR imaging approximately 2 weeks before intra-arterial treatment (mean 16 days, range
0-40) using a 1.5-T MRI unit (Magnetom Avanto, Siemens Medical Solutions, Forchheim, Germany), using our institutional protocol as previously described [15]. For each patient, the sagittal abdominal diameter at the level of the portal vein bifurcation was measured on axial T1-weighted MR images in the portal-venous phase.

\section{C-arm imaging system}

The differences between the two platforms are new acquisition software protocols for DF and DSA that lower the X-ray flux, reducing the dose at the cost of decreased image quality, and a workstation with real-time image processing algorithms to recover for the loss in image quality. In detail, the new acquisition parameters consisted of a decreased tube voltage (75 vs $78 \mathrm{kVp}$ ), use of the smaller focal spot size (0.4 vs. $0.7 \mathrm{~mm}$ ), additional $0.1 \mathrm{~mm}$ copper and $1 \mathrm{~mm}$ aluminium filters. Automatic tube current modulation was enabled on both imaging platforms. The real-time image processing algorithms were spatial noise reduction for DF and spatial noise reduction, temporal averaging and automatic pixel shift for DSA [12].

Besides the new acquisition protocols and the real-time post processing, the two platforms are identical, using a dynamic 14-bit flat panel digital detector with an image matrix of $2480 \times 1920$ pixels, a pixel pitch of $154 \times 154 \mu \mathrm{m}$ and a maximum field of view of $30 \times 38 \mathrm{~cm}$.

The dose-saving features apply only to $2 \mathrm{D}$ imaging and so for $\mathrm{CBCT}$, the acquisition parameters and the 3D reconstruction were identical for both $\mathrm{C}$-arm imaging platforms. The acquisition parameters for $\mathrm{CBCT}$ were $120 \mathrm{kVp}$ tube voltage, $5 \mathrm{~ms}$ exposure and $50 \mathrm{~mA}$ tube current, the last of these being modulated automatically during the acquisition. During $5.2 \mathrm{~s}$ of exposure, 312 projection images ( 60 frames/s) were acquired with the motorized C-arm covering a $240^{\circ}$ clockwise arc at a rotation speed of up to $55 \%$.

\section{TACE protocol}

The indication for treatment and the choice of treatment modality were discussed at our multidisciplinary liver tumor board on a case-by-case basis. All TACE procedures were performed by the same interventional radiologist (JFG) with 18 years of experience in hepatic interventions. A standardized approach according to our institutional protocol was used [16].

A dual-phase C-arm contrast-enhanced CBCT was acquired with the microcatheter placed in the hepatic artery branch considered to be the main tumour feeding vessel to verify correct positioning. After drug delivery, a single CBCT acquisition was performed to confirm technical success of embolization. Additional details of the CBCT protocols for identification of feedings arteries and assessment of treatment 
success have been reported previously $[14,15,17,18]$. CBCTs were not acquired for a few patients who were unable to hold their breath since acquiring $\mathrm{CBCTs}$ with breathing motion would result in insufficient image quality and would have been of no clinical value.

\section{Radiation exposure measurements and calculations}

The new imaging platform (used for the study group) supported Digital Imaging and Communications in Medicine (DICOM) Radiation Dose Structured Reports (RDSR). RDSR contains detailed log information of every X-ray event, including radiation time, air kerma (AK), dose area product (DAP) and number of images acquired (see Appendix 1). A dedicated workstation was set up with DoseUtility (PixelMed Publishing, Bangor, PA) to receive, store and evaluate the RDSRs of the study group patients.

The old imaging platform (used for the control group) did not support RDSR, thus the examination reports generated by the system were used (see Appendix 2). These examination reports contained the $\mathrm{AK}$ and the $\mathrm{DAP}$ of the whole procedure as well as the cumulative DAP and radiation time of all DF runs. However, the DAP of all DSA and CBCT runs was combined into a single "Cumulative DAP (exposure)" on these examination reports (see Appendix 2). To separate DAP of DSA and CBCT, the DAP shown on-screen during the treatment before and after each CBCT scan was manually recorded. The DAP of each CBCT was calculated using the following formula:

\section{$\mathrm{DAP}(\mathrm{CBCT})=\mathrm{DAP}($ post $\mathrm{CBCT})-\mathrm{DAP}($ pre $\mathrm{CBCT})$}

The cumulative DAP of all DSA runs was calculated using the formula:

Cumulative DAP(DSA)

$$
=\text { Cumulative } \mathrm{DAP}(\text { exposure })-\text { Cumulative } \mathrm{DAP}(\mathrm{CBCT})
$$

In addition, the examination reports provided only the number of images acquired and not the radiation time for DSA. Thus, the latter had to be calculated using the number of images acquired during each run and knowing the frame rate used. For example, 30 frames at a frame rate of 3 frames per second correspond to a radiation time of $10 \mathrm{~s}$. To prove that all these calculations were correct, examination reports of five patients undergoing TACE on the new system were also collected, and the calculated values were found to be the same as the values recorded using RDSR.

To compensate for the differences in procedure complexity and thus in radiation time between the patients, the recorded and calculated DAP values were normalized to time by dividing by the corresponding radiation times for both DF and DSA runs. For example, the normalized DAP for $1 \mathrm{~min}$ of DF was calculated as DF Cumulative DAP(Gy $\left.\mathrm{cm}^{2}\right) / \mathrm{DF}$ radiation time(min).

\section{DF image quality assessment}

Objective DF image quality assessment was performed on an Osirix workstation (Pixmeo, Bernex, Switzerland) by an interventional radiologist (RES) with more than 4 years of clinical experience in liver imaging and TACE, who did not participate in the TACE procedures. For the assessment, a region of interest (ROI) with an area of $3 \mathrm{~cm}^{2}$ was placed in the abdomen, avoiding gas-filled intestines and bones, and the mean signal intensity and the standard deviation in Hounsfield units (HU) were recorded. A signal-to-noise ratio (SNR) was calculated using the formula $\mathrm{SNR}=$ meanHU/standarddeviation $\mathrm{HU}$. In addition, the signal intensity of the guidance wire in HU was recorded and a contrast ratio (CR) was calculated with the formula $\mathrm{CR}=$ mean $\mathrm{HU} /$ guidance wire $\mathrm{HU}$.

\section{DSA image quality assessment}

Qualitative DSA image analysis was performed by two interventional radiologists (RES and RD), each with more than 4 years of clinical experience in liver imaging and TACE, who did not participate in the TACE procedures. The DSA images of the celiac run of all patients were presented in a blinded and randomized fashion on an Osirix workstation. The readers were blinded to the imaging $\mathrm{C}$-arm system used and the imaging parameters. The window/level settings used were maintained as the default settings in Osirix. Both readers determined independently in separate reading sessions the visibility of the hepatic arteries using a four-point grading score (Table 1).

\section{Statistical analysis}

All statistical computations were performed in SPSS Statistics 22 (IBM Corp., Armonk, NY). A $p$ value less than 0.05 was considered statistically significant. Descriptive statistics were

Table 1 Four-point grading score for the subjective assessment of DSA image quality

\begin{tabular}{lc}
\hline Score & Description \\
\hline 1 & $\begin{array}{c}\text { Perfect visualization of all hepatic arteries } \\
\text { including small intra-tumoral vessels } \\
\text { Visibility from the proper hepatic artery to } \\
\text { the subsegmental branches } \\
2 \\
\text { Visibility from the proper hepatic artery to } \\
\text { the segmental branches } \\
\text { Visibility from the proper hepatic artery to } \\
\text { the lobar arteries only }\end{array}$ \\
\hline
\end{tabular}


performed to summarize the data. The distribution of all scale variables was assessed with the Shapiro-Wilk test. For scale variables with normal distribution, mean, standard deviation and range were used and an unpaired $t$ test was performed. For scale variables with nonGaussian distribution, median, interquartile range and range were used and a Wilcoxon rank-sum test was performed. For ordinal variables, count and percentage were used and a Wilcoxon rank-sum test was performed. Cohen's kappa was used to calculate interrater reliability.

\section{Results}

\section{Patient demographics}

Patients' baseline characteristics are summarized in Table 2. There was no significant difference between the study and the control groups regarding age $(p=0.71)$, BMI $(p=0.95)$ and sagittal abdominal diameter of the patient at the level of the portal vein bifurcation $(p=0.69)$. Hepatocellular carcinoma was the most common cancer in both groups, tumour burden by means of lesion number and number of affected liver segments were similar $(p=0.49$ and $p=0.34$, respectively).

\section{TACE procedures}

Procedure characteristics are summarized in Table 3. Approximately half of the procedures were for TACE-naïve patients in both cohorts. Conventional TACE was performed in the majority of cases $(56 \%$ and $73 \%$ of the study and control groups, respectively). However, only a minority of procedures were performed in a lobar fashion $(21 \%$ and $15 \%$ of the study and control groups, respectively). The median DF time for study and control groups was $15.6 \mathrm{~min}$ (range 5.6-37.2) and $15.5 \mathrm{~min}$ (range 8.6-54.6), respectively. A median of 6 DSA runs was acquired in the study (range 3-11) and control (range 3-13) groups, respectively. The median DSA time for study and control groups was 71.5 s (range 17.4-167.4) and $63.9 \mathrm{~s}$ (range 24.4-204.2). A median of 3 CBCTs was acquired in the study (range 0-5) and control (range 0-6) groups, respectively. There were no statistically significant differences between the two groups regarding DF time $(p=0.34)$, DSA time $(p=$ $0.66)$ as well as number of DSA and CBCT performed ( $p=$ 0.97 and $p=0.56$, respectively).

\section{Radiation exposure}

A detailed description of DAP and AK in terms of median, interquartile range, minimum and maximum is summarized for the study and the control groups in Table 4, including the
Table 2 Baseline characteristics of the study and the control groups

\begin{tabular}{|c|c|c|c|}
\hline $\begin{array}{l}\text { Characteristic } \\
\text { No. of patients }\end{array}$ & $\begin{array}{l}\text { Study group } \\
52(100)\end{array}$ & $\begin{array}{l}\text { Control group } \\
26(100)\end{array}$ & $p$ value \\
\hline \multicolumn{4}{|l|}{ Sex } \\
\hline Female & $15(29)$ & $5(19)$ & \\
\hline Male & $37(71)$ & $21(81)$ & \\
\hline $\mathrm{Age}^{\mathrm{a}}$ & $60.3 \pm 10.8(33-79)$ & $61.3 \pm 10.5(33-80)$ & 0.71 \\
\hline Body mass index ${ }^{a}$ & $27.5 \pm 5.9(16.0-40.9)$ & $27.4 \pm 6.1(16.0-37.9)$ & 0.95 \\
\hline $\begin{array}{l}\text { Sagittal abdominal diameter at the } \\
\text { level of the portal vein bifurcation }(\mathrm{cm})^{\mathrm{a}}\end{array}$ & $25.6 \pm 4.1(18.2-36.7)$ & $25.2 \pm 4.0(18.3-31.2)$ & 0.69 \\
\hline \multicolumn{4}{|l|}{ Tumour type } \\
\hline Hepatocellular carcinoma & $32(61)$ & $20(77)$ & \\
\hline Intrahepatic cholangiocarcinoma & $3(6)$ & $1(4)$ & \\
\hline Secondary liver cancer & $17(33)$ & $5(19)$ & \\
\hline No. of lesions & & & 0.49 \\
\hline 1 & $13(25)$ & $9(35)$ & \\
\hline 2 & $8(15)$ & $4(15)$ & \\
\hline 3 & $8(15)$ & $4(15)$ & \\
\hline$>3$ & $23(45)$ & $9(35)$ & \\
\hline No. of segments affected & & & 0.34 \\
\hline 1 or 2 & $24(46)$ & $14(54)$ & \\
\hline 3 or 4 & $9(17)$ & $3(12)$ & \\
\hline$>4$ & $19(37)$ & $9(35)$ & \\
\hline
\end{tabular}

Except where indicated, data represent numbers of patients and numbers in parentheses are percentages

${ }^{a}$ Data represented as mean \pm standard deviation (range) 
Table 3 Procedure characteristics of the study and the control groups

\begin{tabular}{|c|c|c|c|}
\hline Characteristic & Study group & Control group & $p$ value \\
\hline Sequence no. of procedure & & & 0.62 \\
\hline 1 & $25(48)$ & $11(42)$ & \\
\hline 2 & $14(27)$ & $7(27)$ & \\
\hline 3 & $5(10)$ & $3(12)$ & \\
\hline$>3$ & $8(15)$ & $5(19)$ & \\
\hline \multicolumn{4}{|l|}{ Type of TACE } \\
\hline Conventional TACE & $29(56)$ & $19(73)$ & \\
\hline Drug-eluting beads TACE & $23(44)$ & $7(27)$ & \\
\hline \multicolumn{4}{|l|}{ Selectivity of TACE } \\
\hline Lobar & $11(21)$ & $4(15)$ & \\
\hline Selective & $16(31)$ & $10(38)$ & \\
\hline Superselective & $25(48)$ & $12(46)$ & \\
\hline Catheter positions for drug delivery & & & 0.69 \\
\hline 1 & $40(77)$ & $19(73)$ & \\
\hline 2 & $12(23)$ & $7(27)$ & \\
\hline DF time $(\min )^{\mathrm{a}}$ & $15.6 ; 9.8(5.6-37.2)$ & $15.5 ; 11.0(8.6-54.6)$ & 0.34 \\
\hline No. of DSA acquisitions ${ }^{\mathrm{a}}$ & $6 ; 3.8(3-11)$ & $6 ; 3.0(3-13)$ & 0.97 \\
\hline DSA time $(\mathrm{s})^{\mathrm{a}}$ & $71.5 ; 48.0(17.4-167.4)$ & $63.9 ; 35.3(24.4-204.2)$ & 0.66 \\
\hline No. of CBCT acquisitions ${ }^{\mathrm{a}}$ & $3 ; 2(0-5)$ & $3 ; 1(0-6)$ & 0.56 \\
\hline
\end{tabular}

Except where indicated, data represent numbers of patients and numbers in parentheses are percentages

${ }^{a}$ Data represented as median; interquartile range (range) values normalized by radiation time. By switching to the new system, the cumulative DAP and the cumulative AK decreased by $66 \%$ (from 395.8 to $132.9 \mathrm{~Gy} \mathrm{~cm}^{2}$ ) and $61 \%$ (from 1.16 to $0.49 \mathrm{~Gy}$ ), respectively. The DAP for DF and for exposure (DSA and CBCT) decreased by $52 \%$ (from 99.6 to $47.8 \mathrm{~Gy} \mathrm{~cm}^{2}$ ) and $70 \%$ (from 272.6 to $81.8 \mathrm{~Gy}^{2}$ ), respectively. The DAP reduction for CBCT was only $15 \%$, whereas for DSA it was $79 \%$ (Fig. 1). After normalizing DF and DSA by the radiation time, the DAP for DF and DSA decreased by $47 \%$ (from 6.4 to $3.4 \mathrm{~Gy} \mathrm{~cm} / \mathrm{min}$ ) and $84 \%$ (from 244.7 to $39.9 \mathrm{~Gy} \mathrm{~cm}^{2} / \mathrm{min}$ ), respectively. In the control group, DF and DSA accounted for $27 \%$ and $61 \%$ of the cumulative DAP, respectively. In the study group, the contribution of DF increased to $38 \%$, whereas the contribution of DSA to the cumulative DAP decreased to $38 \%$. Most importantly, the relative contribution of $\mathrm{CBCT}$ to the cumulative DAP increased from $12 \%$ to $24 \%$ by switching to
Table 4 Radiation exposure for the study and the control groups

\begin{tabular}{|c|c|c|c|c|}
\hline & Study group & Control group & Reduction & $p$ value \\
\hline Cumulative DAP $\left(\mathrm{Gy} \mathrm{cm}^{2}\right)$ & $\begin{array}{l}132.9 ; 146.2 \\
\quad(30.7-588.4)\end{array}$ & $\begin{array}{l}395.8 ; 434.4 \\
\quad(86.2-1469.9)\end{array}$ & $66 \%$ & $<0.01$ \\
\hline Cumulative AK (Gy) & $0.49 ; 0.52(0.06-2.35)$ & $1.16 ; 1.18(0.28-3.82)$ & $61 \%$ & $<0.01$ \\
\hline DF DAP (Gy $\left.\mathrm{cm}^{2}\right)$ & $\begin{array}{l}47.8 ; 62.3 \\
\quad(9.4-288.6)\end{array}$ & $\begin{array}{l}99.6 ; 105.2 \\
\quad(28.3-307.4)\end{array}$ & $52 \%$ & $<0.01$ \\
\hline DF DAP/min (Gy cm²/min) & $3.4 ; 3.7(1.1-15.3)$ & $6.4 ; 5.2(2.2-12.0)$ & $47 \%$ & $<0.01$ \\
\hline Exposure DAP $\left(\mathrm{Gy} \mathrm{cm}^{2}\right)$ & $\begin{array}{l}81.8 ; 79.8 \\
\quad(10.2-380.1)\end{array}$ & $\begin{array}{l}272.6 ; 397.6 \\
\quad(58.0-1197.1)\end{array}$ & $70 \%$ & $<0.01$ \\
\hline $\begin{array}{l}\text { CBCT DAP for } 1 \text { CBCT } \\
\left(\mathrm{Gy} \mathrm{cm}^{2}\right)\end{array}$ & $14.0 ; 7.3(4.7-21.2)$ & $16.5 ; 12.6(5.2-22.8)$ & $15 \%$ & 0.51 \\
\hline $\begin{array}{l}\text { Cumulative CBCT DAP } \\
\left(\mathrm{Gy} \mathrm{cm}^{2}\right)\end{array}$ & $\begin{array}{l}30.5 ; 36.7 \\
\quad(0.0-103.7)\end{array}$ & $44.8 ; 40.7(0.0-82.4)$ & $32 \%$ & 0.19 \\
\hline DSA DAP $\left(\mathrm{Gy} \mathrm{cm}^{2}\right)$ & $\begin{array}{l}48.8 ; 77.6 \\
\quad(3.0-316.7)\end{array}$ & $\begin{array}{l}228.4 ; 371.2 \\
\quad(42.4-1153.0)\end{array}$ & $79 \%$ & $<0.01$ \\
\hline DSA DAP/min $\left(\mathrm{Gy} \mathrm{cm}^{2} / \mathrm{min}\right)$ & $\begin{array}{l}39.9 ; 42.5 \\
\quad(9.5-192.3)\end{array}$ & $\begin{array}{l}244.7 ; 237.9 \\
\quad(62.2-453.9)\end{array}$ & $84 \%$ & $<0.01$ \\
\hline
\end{tabular}

Data represented as median; interquartile range (range) 
Fig. 1 Box plot showing the distribution of radiation exposure (dose area product in $\mathrm{Gy} \mathrm{cm}^{2}$ ) for the study and the control groups for the entire procedure, for digital subtraction angiography, digital fluoroscopy and cone beam CT. The plot uses power scale on the y-axis and shows the interquartile range (box), 5 th and 95th percentiles (outermost bars) and the median (thick horizontal line) of the exposure distribution in each system

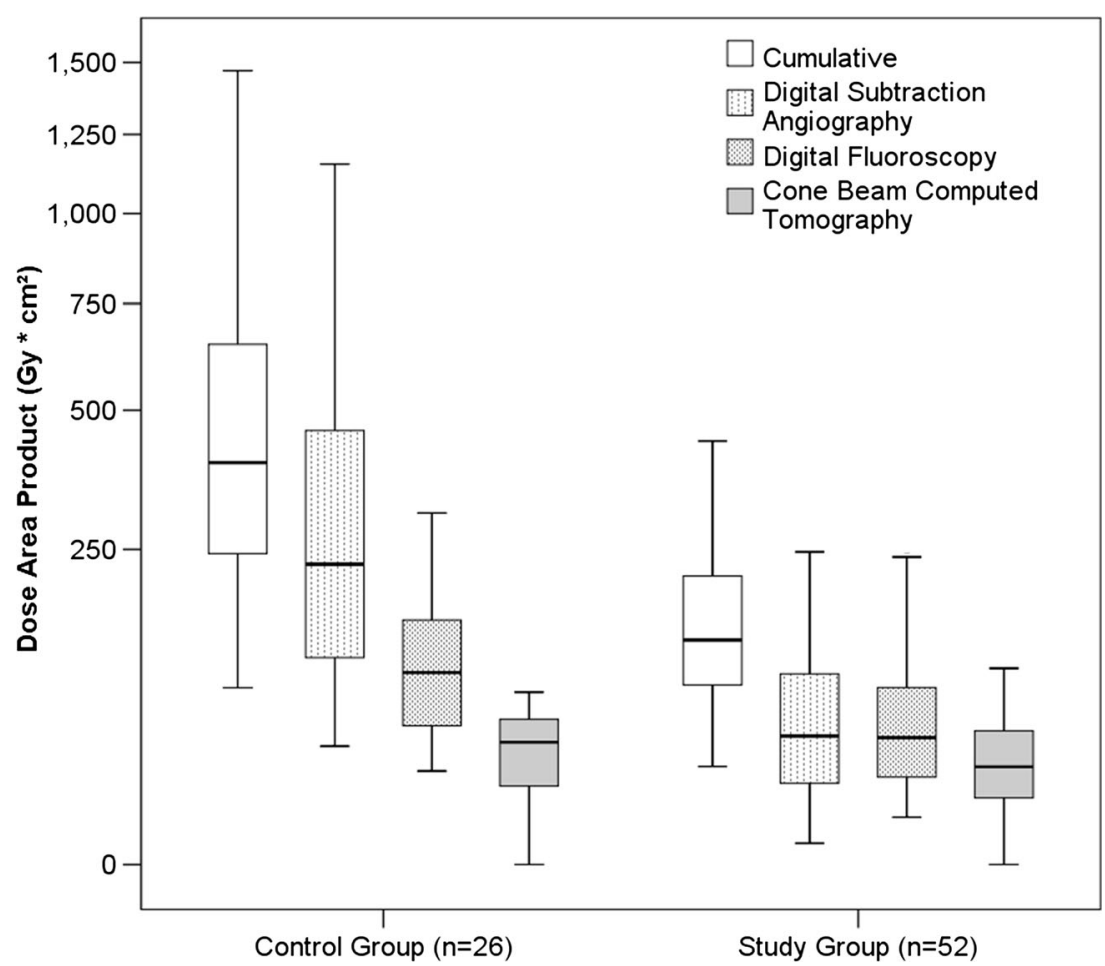

the new system. In other words, the radiation exposure of one CBCT was equivalent to $155 \mathrm{~s}$ DF or $4 \mathrm{~s}$ DSA in the control group and $247 \mathrm{~s}$ DF or $21 \mathrm{~s}$ DSA in the study group.

\section{DF image quality}

The SNR of the new platform was significantly higher than that of the old platform $[31.1 \pm 6.0$ (range $17.7-49.9$ ) vs $23.2 \pm$ 4.4 (range $15.7-31.8$ ); $p<0.01]$. The visibility of the guide wire expressed as $\mathrm{CR}$ on the other hand was significantly lower in the new platform $[1.74 \pm 0.35$ (range $1.27-2.63$ ) vs $2.00 \pm 0.30$ (range 1.46-2.50); $p \leq 0.01]$.

\section{DSA image quality}

There was no statistically significant difference regarding the image quality between the two cohorts according to both readers ( $p=0.82$ and $p=0.75$, respectively) using the provided four-point grading score for the visibility of hepatic arteries. Both readers rated the majority of celiac arteriograms in both cohorts as perfect (category 1) (Fig. 2). No celiac arteriogram was rated as category 4 (Table 5). Cohen's kappa showed a substantial agreement between both readers $(\kappa=0.76$ and $\kappa=$ 0.66 for the old and new platforms, respectively, $p<0.01)$. A detailed distribution of image quality scores is shown in Table 5.
Fig. 2 Celiac arteriogram during TACE in two patients with neuroendocrine tumour. Left panel was acquired on the preceding imaging platform and the right panel on the new imaging platform. Both arteriograms were of diagnostic quality, showing the tumourfeeding arteries and the tumour blush (arrowheads). However, the new imaging platform resulted in a significantly lower radiation exposure during the acquisition of the celiac arteriogram

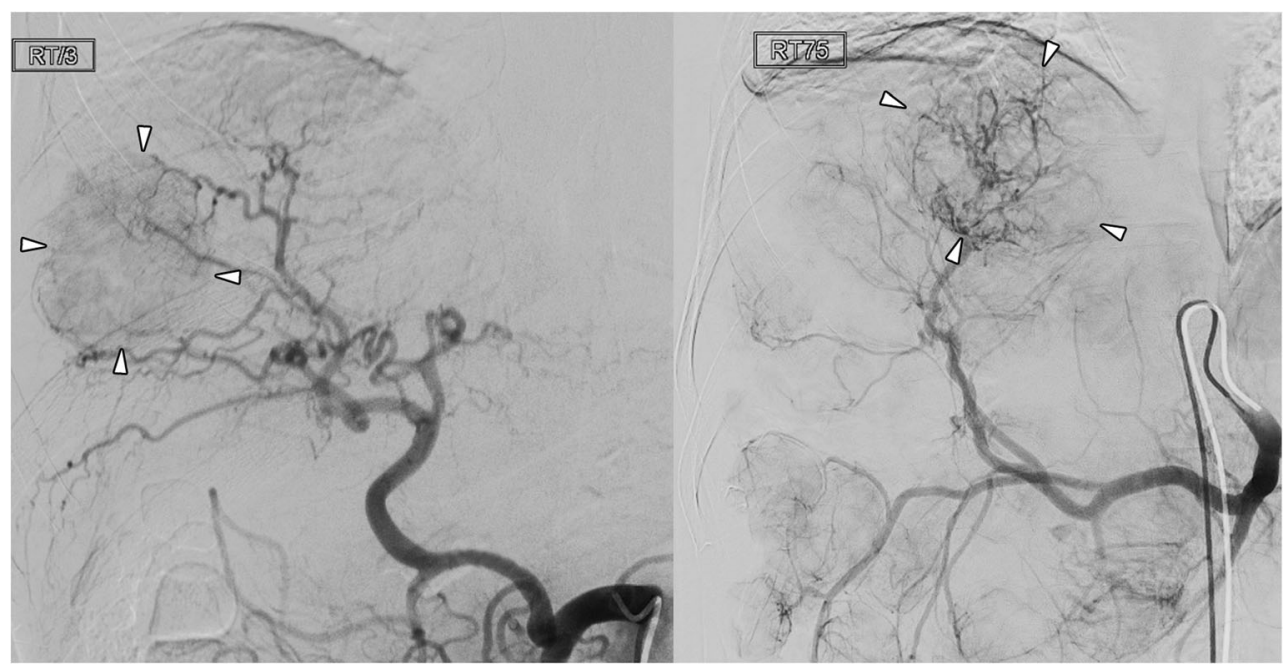


Table 5 DSA Image quality score cross-tables of both readers for the study and the control groups

\begin{tabular}{lrrrrr}
\hline & & \multicolumn{2}{c}{ Reader 1 } & & Total \\
\cline { 3 - 5 } & & 1 & 2 & 3 & \\
\hline Study group & & & & & \\
Reader 2 & 1 & 30 & 4 & 0 & 34 \\
& 2 & 4 & 14 & 0 & 18 \\
& 3 & 0 & 0 & 0 & 0 \\
Total & & 34 & 18 & 0 & 52 \\
Control group & & & & & \\
Reader 2 & 1 & 17 & 2 & 0 & 19 \\
& 2 & 0 & 4 & 0 & 4 \\
Total & 3 & 0 & 1 & 2 & 3 \\
& & 17 & 7 & 2 & 26 \\
\hline
\end{tabular}

\section{Discussion}

The main finding of our study was the significant radiation exposure reduction of up to $66 \%$ for the entire TACE procedure as a result of the new imaging platform. This significant reduction in radiation exposure is especially important in patients being treated repeatedly with TACE, in similar therapies such as radio-embolization, or as a bridging or downstaging prior to liver transplantation [19]. Furthermore, the reduction in radiation exposure using the new system is also of importance for the in-room medical staff, because they are exposed to radiation on a regular basis.

Of note, the radiation exposure reduction was only significant for two-dimensional runs (such as DF and DSA) and not for $\mathrm{CBCT}$ acquisitions. This was expected as the dose reduction techniques in our work apply for 2D imaging only. The DSA runs showed the highest radiation exposure reduction of up to $84 \%$, and the exposure during DF and CBCT runs decreased by $47 \%$ and $15 \%$, respectively. Thus, the relative impact of DSA on the cumulative DAP decreased from $61 \%$ to $38 \%$, and the relative impact of $\mathrm{DF}$ and $\mathrm{CBCT}$ increased from 27 to $38 \%$ and from $12 \%$ to $24 \%$, respectively. Given the steady increase in the use of CBCT in TACE procedures because of its proven benefits [20-26], dose reduction for this imaging modality should be the next logical step to decrease cumulative radiation exposure even further.

Another important finding was that the image quality of DSA runs by means of subjective assessment of hepatic vessel visualization was not affected by the significant reduction of radiation exposure. For DF runs, the new platform showed a significantly improved SNR at the cost of a significantly decreased CR, in comparison to the old platform. However, the DF radiation time did not increase, clearly showing that this objectively measured difference in DF image quality did not have a negative impact on the course of TACE procedures. In other words, the image quality was still sufficient to continue the TACE procedure.

Comparison with other studies using absolute numbers is difficult because different metrics were used. Paul et al. [27] used peak skin dose and DAP to compare three generations of angiographic imaging systems. These authors reported a radiation exposure reduction of $62 \%, 15 \%$ and $49 \%$ for DF, DSA and CBCT during TACE procedures, respectively. The main reason for the significant reduction of CBCT DAP in their study was a faster CBCT acquisition protocol ( 3 vs. $8 \mathrm{~s}$ ), which also resulted in fewer projection images being acquired (133 vs. 382 frames). However, their mean DAP for a CBCT run was $21.58 \mathrm{~Gy} \mathrm{~cm}^{2}$, whereas our CBCT protocol resulted in a median DAP of $13.9 \mathrm{~Gy} \mathrm{~cm}^{2}$ with $5.2 \mathrm{~s}$ of radiation time (the same acquisition protocol was used for both control and study imaging platforms). In addition, most studies do not normalize their exposure measurements by the radiation time, but only report radiation exposure and time as separate values. In our study, radiation exposure and time values were used together to calculate DF and DSA DAP for 1 min of radiation time each to normalize for differences in procedure complexity and to enable a better comparison with other studies. We report a median DF and DSA DAP per minute of 3.4 and $39.9 \mathrm{~Gy} \mathrm{~cm}^{2}$ on the new imaging platform, respectively. Calculating these normalized values for the previously mentioned study [27], their mean DF and DSA DAP were 5.8 and $92.9 \mathrm{~Gy}^{2}$ on the latest generation of imaging platform used, respectively. This underlines the substantial radiation exposure reduction achieved by the new imaging platform used in our study.

Our study had several limitations. First, the number of patients included in the control group was limited to 26 by the upgrade of the imaging platform. However, even with a relatively small sample size of 78 patients, a statistically significant radiation exposure reduction was achieved while the study and the control groups did not show any significant differences in patient characteristics and in radiation time. Second, RDSR was not available for the preceding imaging platform, thus manual exposure logging had to be performed for all patients undergoing TACE on that platform and the DSA radiation time was calculated retrospectively using the number of images acquired during each DSA run. However, these calculations were verified by comparison to RDSR for a subset of patients examined on the new imaging platform.

In conclusion, the new imaging platform significantly reduced radiation exposure for TACE procedures without increased radiation time or negative impact on image quality.

Acknowledgments The scientific guarantor of this publication is JeanFrancois Geschwind, MD. The authors of this manuscript declare relationships with the following companies: Jean-Francois Geschwind, MD - Consultant for Biocompatibles/BTG, Bayer HealthCare, Guerbet, Nordion/BTG, Philips Healthcare and Jennerex. MingDe Lin, PhDPhilips employee 
Our study has received funding by the Max Kade Foundation, Inc., NY, USA, NIH/NCI R01 CA160771, P30 CA006973, Philips Research North America, Briarcliff Manor, NY, USA. One of the authors (RES) has significant statistical expertise. Institutional review board approval was obtained. Written informed consent was waived by the institutional review board. Methodology: prospective, observational, performed at one institution.

Open Access This article is distributed under the terms of the Creative Commons Attribution-NonCommercial 4.0 International License (http:// creativecommons.org/licenses/by-nc/4.0/), which permits any noncommercial use, distribution, and reproduction in any medium, provided you give appropriate credit to the original author(s) and the source, provide a link to the Creative Commons license, and indicate if changes were made.

\section{References}

1. Mettler FA Jr, Bhargavan M, Faulkner K et al (2009) Radiologic and nuclear medicine studies in the United States and worldwide: frequency, radiation dose, and comparison with other radiation sources-1950-2007. Radiology 253(2):520-531

2. Berrington de Gonzalez A, Darby S (2004) Risk of cancer from diagnostic X-rays: estimates for the UK and 14 other countries. Lancet 363(9406):345-351

3. Shope TB (1996) Radiation-induced skin injuries from fluoroscopy. Radiographics 16(5):1195-1199

4. Koenig TR, Mettler FA, Wagner LK (2001) Skin injuries from fluoroscopically guided procedures: part 2, review of 73 cases and recommendations for minimizing dose delivered to patient. AJR Am J Roentgenol 177(1):13-20

5. Preston RJ (2005) Radiation biology: concepts for radiation protection. Health Phys 88(6):545-556

6. Lencioni R, Petruzzi P, Crocetti L (2013) Chemoembolization of hepatocellular carcinoma. Semin Interv Radiol 30(1):3-11

7. Kuhlmann JB, Blum HE (2013) Locoregional therapy for cholangiocarcinoma. Curr Opin Gastroenterol 29(3):324-328

8. Fiorentini G, Aliberti C, Mulazzani L et al (2014) Chemoembolization in colorectal liver metastases: the rebirth. Anticancer Res 34(2):575-584

9. Del Prete M, Fiore F, Modica R et al (2014) Hepatic arterial embolization in patients with neuroendocrine tumors. J Exp Clin Cancer Res 33(1):43

10. Sato T (2010) Locoregional management of hepatic metastasis from primary uveal melanoma. Semin Oncol 37(2):127-138

11. Miller DL, Balter S, Cole PE et al (2003) Radiation doses in interventional radiology procedures: the RAD-IR study: part I: overall measures of dose. J Vasc Interv Radiol 14(6):711-727

12. Soderman M, Holmin S, Andersson T, Palmgren C, Babic D, Hoornaert B (2013) Image noise reduction algorithm for digital subtraction angiography: clinical results. Radiology 269(2):553560

13. Soderman M, Mauti M, Boon S et al (2013) Radiation dose in neuroangiography using image noise reduction technology: a population study based on 614 patients. Neuroradiology 55(11): $1365-1372$

14. Tacher V, Radaelli A, Lin M, Geschwind JF (2015) How I do it: cone-beam CT during transarterial chemoembolization for liver cancer. Radiology 274(2):320-334

15. Schernthaner RE, Lin M, Duran R, Chapiro J, Wang Z, Geschwind JF (2014) Delayed-phase cone-beam CT improves detectability of intrahepatic cholangiocarcinoma during conventional transarterial chemoembolization. Cardiovasc Intervent Radiol. doi:10.1007/ s00270-014-1026-7

16. Liapi E, Geschwind JF (2011) Transcatheter arterial chemoembolization for liver cancer: is it time to distinguish conventional from drug-eluting chemoembolization? Cardiovasc Intervent Radiol 34(1):37-49

17. Loffroy R, Lin M, Yenokyan G et al (2013) Intraprocedural C-arm dual-phase cone-beam CT: can it be used to predict short-term response to TACE with drug-eluting beads in patients with hepatocellular carcinoma? Radiology 266(2):636-648

18. Wang Z, Lin M, Lesage D et al (2014) Three-dimensional evaluation of lipiodol retention in HCC after chemoembolization: a quantitative comparison between CBCT and MDCT. Acad Radiol 21(3): 393-399

19. Fujiki M, Aucejo F, Choi M, Kim R (2014) Neo-adjuvant therapy for hepatocellular carcinoma before liver transplantation: where do we stand? World J Gastroenterol 20(18):5308-5319

20. Kakeda S, Korogi Y, Ohnari N et al (2007) Usefulness of conebeam volume CT with flat panel detectors in conjunction with catheter angiography for transcatheter arterial embolization. J Vasc Interv Radiol 18(12):1508-1516

21. Miyayama S, Yamashiro M, Hattori Y et al (2011) Efficacy of conebeam computed tomography during transcatheter arterial chemoembolization for hepatocellular carcinoma. Jpn J Radiol 29(6):371-377

22. Miyayama S, Yamashiro M, Okuda M et al (2009) Usefulness of cone-beam computed tomography during ultraselective transcatheter arterial chemoembolization for small hepatocellular carcinomas that cannot be demonstrated on angiography. Cardiovasc Intervent Radiol 32(2):255-264

23. Meyer BC, Frericks BB, Voges M et al (2008) Visualization of hypervascular liver lesions during TACE: comparison of angiographic C-arm CT and MDCT. AJR Am J Roentgenol 190(4): W263-W269

24. Loffroy R, Favelier S, Cherblanc V, Estivalet L (2013) C-arm dualphase cone-beam CT: a revolutionary real-time imaging modality to assess drug-eluting beads TACE success in liver cancer patients. Quant Imaging Med Surg 3(4):196-199

25. Wallace MJ (2007) C-arm computed tomography for guiding hepatic vascular interventions. Tech Vasc Interv Radiol 10(1):79-86

26. Iwazawa J, Ohue S, Hashimoto N, Muramoto O, Mitani T (2012) Survival after C-arm CT-assisted chemoembolization of unresectable hepatocellular carcinoma. Eur J Radiol 81(12):39853992

27. Paul J, Jacobi V, Farhang M, Bazrafshan B, Vogl TJ, Mbalisike EC (2013) Radiation dose and image quality of X-ray volume imaging systems: cone-beam computed tomography, digital subtraction angiography and digital fluoroscopy. Eur Radiol 23(6):1582-1593 